\title{
Physio-behavioral Coupling as an Index of Team Processes and Performance: Overview, Measurement, and Empirical Application
}

\author{
Adam J. Strang ${ }^{1}$, Gregory J. Funke ${ }^{2}$, Sheldon M. Russell ${ }^{2}$, and Robin D. Thomas ${ }^{3}$ \\ ${ }^{1}$ Consortium Research Fellows Program, Alexandria VA, USA \\ ${ }^{2}$ Air Force Research Laboratory, Wright-Patterson AFB OH, USA \\ ${ }^{3}$ Miami University, Oxford OH, USA \\ adam.strang.ctr@us.af.mil
}

\begin{abstract}
Research shows that teammates often exhibit similarity in their physiological and behavioral responses during cooperative task performance, a phenomenon referred to here as physio-behavioral coupling (PBC). Goals of this manuscript are to provide an overview of research examining the utility of $\mathrm{PBC}$ as an index of team processes (e.g., coordination) and performance, discuss applied and theoretical issues in PBC measurement, and present findings from a study using linear and nonlinear statistics to assess PBC.
\end{abstract}

Keywords: Team, Coupling, Coordination, Performance, Nonlinear.

\section{Introduction}

This manuscript supports the parallel session entitled, "Modeling the Complex Dynamics of Teamwork." The focus of this manuscript is physio-behavioral coupling (PBC), its relation to team processes and performance, and issues regarding its measurement and interpretation, with an emphasis on practical applications. An experimental illustration of these issues is provided.

\subsection{PBC, Team Processes, and Team Performance}

PBC can be defined as a statistical similarity in the cortical, autonomic, or behavioral activity of two or more members of a team engaged in cooperative behavior. Over the past three decades, researchers have identified PBC in a number of physio-behavioral responses, including cardiac inter-beat intervals (IBIs), electrical brain activity, and human postural sway, and in diverse team task environments, such as military room clearing, team puzzle solving, and duet guitar playing [1-5]. In many cases, PBC manifests as an emergent (i.e., spontaneous and selforganized) phenomenon outside conscious awareness [1]. As such, there has been speculation about the underlying causes (or drivers) of PBC, its role in cooperative task performance, and the associations it shares with important team processes (e.g., strategy, coordination, communication, cohesion, etc.). 
Most explanations posit that PBC reflects important team-level processes, such as communication and coordination [1]. For example, oral communication is a vector employed by teams to discuss strategy and coordinate action [6]. Research has demonstrated that oral communication is sufficient to drive the coupling of human postural sway, supporting speculation that sway coupling can serve as an indirect index of team communication dynamics [4] - a speculation that our own research supports [5]. Other research has identified $\mathrm{PBC}$ between group members performing very different actions (e.g., active participants and passive observers) [7], supporting the perspective that PBC may be caused by emotional (arousal) and/or cognitive (shared situation awareness) dynamics associated with group/team membership. Finally, PBC has been shown to exhibit relationships with psychosocial phenomenon like rapport and trust $[8,9]$, prompting some to speculate that $\mathrm{PBC}$ may facilitate, rather than simply reflect a consequence of, team processes.

To date, only a small number of studies have examined the association between PBC and team performance. While several studies suggest a positive relationship (i.e., higher $\mathrm{PBC}$ is related to better team performance) [2, 3], our research indicates that a negative relationship is possible [5].

From an applied perspective, PBC has been shown to exhibit a moderate relationship with performance (absolute $r \sim .4$ ) $[1,5]$, which is comparable in magnitude to correlations observed between performance and other team processes such as cohesion and collective efficacy (both $r \sim .25$ ) [10, 11]. This suggests that PBC measures may have an advantage over other (largely self-reported) team process assessments since many responses used to estimate PBC are minimally invasive (e.g., cardiac IBI, postural sway), and many metrics of $\mathrm{PBC}$ can be computed in real-time without interrupting task performance.

\subsection{PBC Measurement: A Historical Review and Recent Developments}

PBC has been characterized using a variety of different statistical measures, leading to inconsistency across studies. It has also been exceedingly rare for researchers to communicate why a particular measure (or set of measures) was chosen to over others (see [7] and [12] for exceptions). However, choosing the proper measure is critical since it may influence the ability to detect meaningful changes in PBC, determine the information about PBC obtained (e.g., coupling strength versus phase relation), and have implications for the utility of PBC measurement in applied applications.

Early PBC studies used independent rater analysis of recorded video and/or physiological signals to detect response similarities [8]. While these methods were carefully implemented, they are subjective, as well as both cost and time prohibitive.

In more recent studies assessing PBC in cyclical motor tasks (e.g., swinging of handheld pendulums), researchers have often employed relative phase statistics [13]. Though relative phase is an intuitive indicator of synchronicity (a specific sub-type of coupling) and phase relation (e.g., in-phase versus out-of-phase), it is effective primarily for examining responses that exhibits near-sinusoidal oscillations [14].

An additional approach employed by Henning and colleagues [2] has been to examine PBC using cross-correlation (CC) and cross-spectral coherence (CSC), which 
are linear statistics that describe the degree of similarity between two time series in the time and frequency domains, respectively. Advantages of the measures include a long and accepted history for examining complex time-series data and the ability to provide estimates of multiple coupling dynamics (CC: coupling strength and temporal lag; CSC: coupling strength at particular frequencies) in near-real-time [15]. Disadvantages include linear assumptions of periodicity and stationarity (i.e., equal mean and variance), which many physio-behavioral responses are known to violate $[16,17]$.

In an attempt to overcome the limitations of linear statistics, some researchers (including the current authors) have explored the use of nonlinear measures to characterize PBC. Although computationally quite different from one another, this family of statistics, which include measures such as Cross-Recurrence Quantification Analysis (CRQA) [12], Cross Sample Entropy (CSEn) [18], and Average Mutual Information (AMI) [19], can be used to confirm the existence of nonlinear coupling and quantify its strength. To illustrate how the information obtained from linear and nonlinear coupling measures differ, consider that $\mathrm{CC}$, when a zero lag is employed, is equivalent to a Pearson product-moment correlation [20]. Thus, CC characterizes the degree to which two time-series share a one-to-one (i.e., linearly synchronized) relationship in both time and (relative) amplitude. Conversely, nonlinear measures (with acknowledgment that the following is a broad generalization) quantify the degree to which two time series exhibit matching temporal patterns (i.e., strings of sequential data points) across an entire time interval, regardless of where those matches occur within that interval. Thus, nonlinear measures do not index synchronicity (a potential limitation if this is the coupling dynamic of specific interest), but rather the overall degree of patterning shared between two data streams within a specified temporal envelope.

It is because of this flexibility that nonlinear measures may be better suited for detecting and quantifying coupling strength in aperiodic and noisy systems [12]. This view is supported by findings that nonlinear coupling measures demonstrate greater sensitivity, compared to more traditional linear measures like CC and CSC, for detecting changes in coupling dynamics among paired physical systems [21], financial trends [22], human postural sway [12], and animal neurophysiological responses [23].

However, claiming that nonlinear coupling measures are more sensitive than linear measures, without first identifying that the systems under examination exhibit meaningful (i.e., deterministic) linear and/or nonlinear coupling, is problematic. To establish that meaningful coupling is evident, surrogation tests are required.

The most straightforward and intuitive method to perform surrogation tests first involves obtaining estimates of PBC (for each metric of interest) from originally sampled time-series representing the response(s) of interest (e.g., postural sway from two people engaged in oral communication). Next, new (surrogate) time-series are generated by (separately) randomly shuffling the sequence of data points within each original time-series. The result of this procedure are two time-series in which any deterministic temporal structure that originally existed in individual responses, as well as any meaningful coupling between those responses, is eliminated. Then, PBC estimates are obtained for the surrogate time-series and compared with those from the original time-series. If the two sets of PBC estimates are shown to be equivalent (often determined using inferential statistics applied to an entire experiment's sample), this suggests that no meaningful coupling existed in the original time-series. Conversely, if $\mathrm{PBC}$ estimates in the original time-series are greater than those observed 
from the surrogates, this suggests that meaningful coupling does exist in the original. In a case where both linear and nonlinear measures are used to examine PBC, and both detect meaningful coupling, then it is possible to examine the PBC metrics for sensitivity differences, with the understood caveat that each type of metric characterizes a different coupling dynamic. However, in a case where meaningful nonlinear coupling is detected but linear coupling is not, investigating sensitivity differences is futile, since the very application of linear statistics in this case is inappropriate.

To date, very few PBC studies have included any form of surrogation test. However, it is our view that these tests are critical since they not only provide useful information about the underlying dynamics of a coupled relationship, but also verify the appropriateness of statistics used to draw inference about the phenomenon.

\section{Empirical Application of Linear and Nonlinear Measures for Assessing PBC in a Cooperative Team Task}

The remainder of this manuscript is dedicated to describing methods and results from a single experiment in which PBC was examined in dyads performing a cooperative pointing task (Fig. 1). The purpose of including this experiment here is to provide guidance on application of linear and nonlinear measures to examine PBC, as well as explore the unique (or analogous) information linear and nonlinear metrics may provide about team coordination and performance.

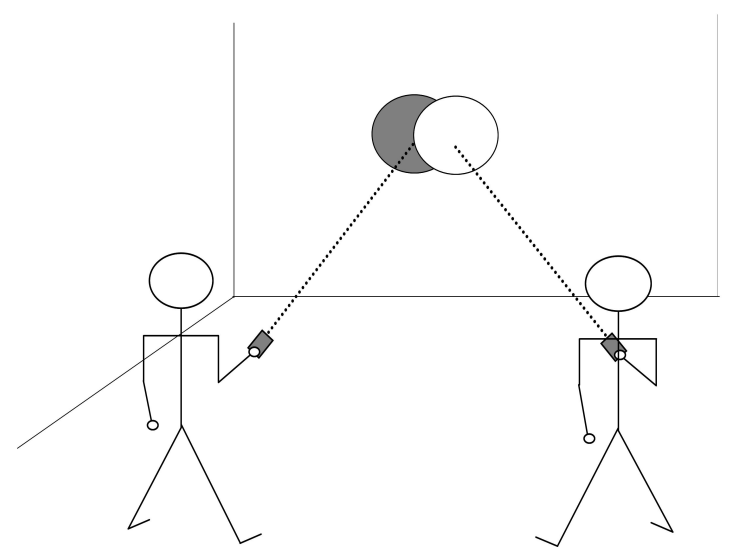

Fig. 1. Illustration of the cooperative pointing task used in this experiment. The goal of the task was to achieve complete overlap of two "virtual" circles projected onto a far wall by manipulating handheld remote controls (Nintendo Wii Remotes) that controlled the circles' movements.

The pointing task employed in this experiment required dyads to control the movements of two "virtual" circles (each participant controlled one circle) and align them such that the two circles completely overlapped one another. This particular task was selected because it presented a context in which PBC (i.e., wrist coupling), team coordination, and team performance should be closely related. Thus, we hoped in 
utilizing this task we might draw more direct inference from PBC measurement about the coordination dynamics supporting team performance, and about how those dynamics are altered by a manipulation of task constraints (i.e., an increase in difficulty). Other goals included a determination of the presence of meaningful linear and/or nonlinear coupling in team members' wrist movements (using surrogation tests) and a comparison of the sensitivity of linear and nonlinear coupling measures to team performance differences across task difficulty conditions.

\subsection{Methods}

To perform the pointing task, dyads $(N=30$ pairs $)$ stood upright facing a far wall at a distance of $1.5 \mathrm{~m}$ while holding handheld remote controls (Nintendo Wii remotes "wiimotes") in their dominant hand with elbow flexed to 90 degrees. Two large circles (145 and 150 pixels in diameter, respectively) were shown on a far wall using a video projector. The vertical and lateral movements of the circles were linked to the movements of the wiimotes using custom software and Bluetooth connection. The task performance goal was to achieve complete overlap of the two circles (at a location near the center of the wall) and hold that position for "as long as possible" in 90 second trials.

Task difficulty was manipulated by altering the wiimote-to-circle movement ratio. In the easy condition the ratio was $1: .25$, meaning that a $1 \mathrm{~cm}$ translation of the wiimote elicited a $.25 \mathrm{~cm}$ translation of the circle to which it corresponded. In the normal and hard conditions the ratio was $1: 1$ and $3: 1$, respectively.

Dyads performed two trials of each condition in counterbalance order (six trials total). Throughout trials wrist movements (yaw and pitch rotation) were recorded from participants at $75 \mathrm{~Hz}$ using two wireless Xsens Technologies Mtw inertial trackers. In post-processing, yaw and pitch time-series were cropped to 60 second durations by removing the first and last 15 seconds of each trial. The truncated times-series were then subjected to .1 to $30 \mathrm{~Hz} 2^{\text {nd }}$ order Band-pass Butterworth filters (to achieve stationarity and eliminate high frequency noise) and normalized to unit variance.

Normalized time-series were then paired within dyad and rotational plane and examined using CC, CRQA (percent recurrence; \%REC) ${ }^{1}, \mathrm{CSEn}^{2}$ and AMI in $13.65 \mathrm{sec}$ (1,024 data point) windows with a 6.83 (512 point) overlap. ${ }^{3}$ This procedure rendered six values in each trial for each PBC measure; from those six values, the median was recorded for each measure to indicate the central tendency of yaw and pitch wrist coupling. Median PBC estimates from like conditions were then averaged.

\footnotetext{
$1 \%$ REC is the percentage of points (where point represents a distance vector comprised of a serial sequences of data values) that repeat in a 2-dimenstional recurrence plot. It serves as an indicant of the overall amount of patterning in a time-series [12].

${ }^{2}$ In subsequent reporting the inverse of CSEn, $\mathrm{CSEn}^{-1}$, is presented to facilitate directional correspondence with interpretation of all other PBC measures.

${ }^{3} \mathrm{CC}$ was estimated with zero lag, replicating the procedures of [2]. CSEn parameters, $M=3$ (vector lengths for comparison) and $r=.3$ (vector tolerance) were set using a parameter selection procedure described by [24]. CRQA parameters, i.e., embedding dimension $(E m D=9)$, time delay $(t d=4)$, rescaling method (rescale $=$ euclidean $)$, and radius $(\mathrm{rad}=10)$, were established using the procedure described by [12]. AMI requires no parameter selection using the algorithm provided in [19].
} 


\section{$2.2 \quad$ Results}

Surrogation tests were used to determine whether meaningful linear and/or nonlinear coupling existed between wrist movements of dyads using identical methods to those described earlier in section 1.2. Inferential comparisons testing for differences in PBC estimate from original and surrogate time-series were carried out using paired samples $t$-tests for all PBC metrics.

Results indicated that, across nonlinear measures, PBC estimates from the original time-series were significantly greater than those obtained from surrogates. However, no difference was found between PBC estimates of original and surrogate time-series for CC (Table 1). This indicates that the wrist movements of teammates exhibited a nonlinear, as opposed to linear, coupled relationship. From a practical perspective this means that dyad wrist movements did not exhibit linear synchronicity, though they did exhibit meaningful similarities in overall temporal patterning. Consequently, CC was dropped from further analyses and comparisons.

Table 1. Mean of median PBC estimates and standard errors (in parentheses) obtained from original and surrogate (randomly shuffled) time-series pairings

\begin{tabular}{lccc}
\hline PBC Measure & Original pairs & Surrogate pairs & $t$ \\
\hline CC - yaw & $-.01(.01)$ & $.00(.00)$ & .53 \\
CC - pitch & $-.03(.01)$ & $.00(.00)$ & 1.87 \\
CSEn - yaw & $11.01(.25)$ & $.68(.00)$ & $41.40^{*}$ \\
CSEn - pitch & $10.76(.33)$ & $.67(.00)$ & $30.14^{*}$ \\
\%REC - yaw & $3.95(.13)$ & $.00(.00)$ & $30.39^{\star}$ \\
\%REC - pitch & $3.83(.13)$ & $.00(.00)$ & $29.71^{*}$ \\
AMI - yaw & $.29(.00)$ & $.03(.00)$ & $56.64^{*}$ \\
AMI - pitch & $.30(.05)$ & $.03(.00)$ & $55.32^{*}$ \\
\hline
\end{tabular}

Note. $t$-crit $d f=89, \alpha=.05=1.99$.

$* p<.05$

Next, effects of task difficulty were examined using separate repeated-measures ANOVAs for PBC measures and the team performance metric, Circle Overlap ${ }^{4}$. Omnibus main effects of Circle Overlap, $F(1.60,46.31)=2701.14, p<.05$, and AMIyaw, $F(1.87,54.33)=6.89, p<.05$, were detected. Post-hoc pairwise comparisons revealed a precipitous decline in Circle Overlap as a product of increases in task difficulty (Fig. 2a). Circle Overlap decreased as task difficulty increased, which confirms that the experimental manipulation was effective in diminishing team performance.

Post-hoc assessment of AMI-yaw revealed lower wrist coupling in the normal and hard, as compared to the easy condition - indicating that nonlinear wrist coupling decreased as a result of increases in task difficulty (Fig. 2b).

\footnotetext{
${ }^{4}$ Circle Overlap is defined as the cumulative time (in seconds) during a 90 second trial that complete overlap of the two circles was achieved.
} 

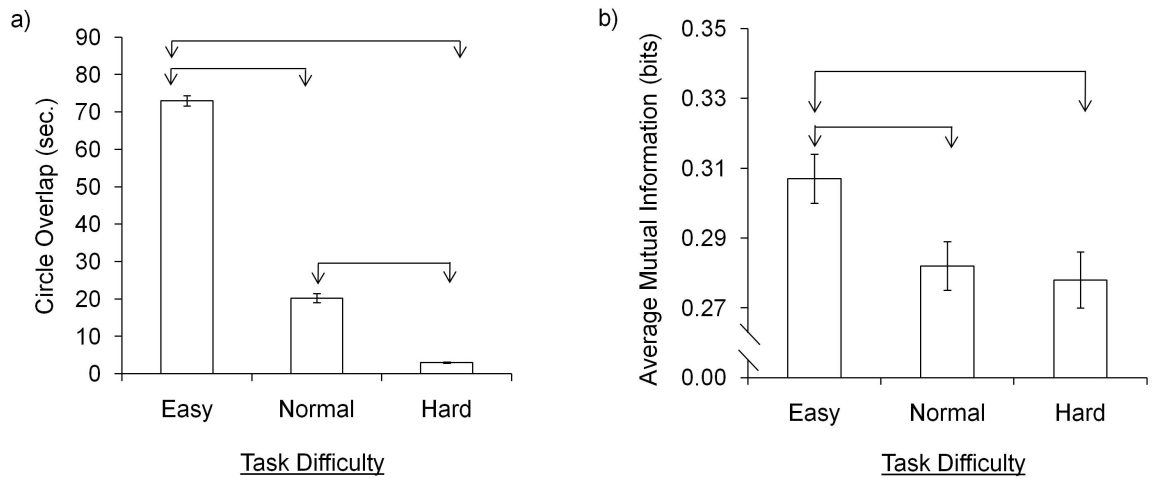

Fig. 2. Mean estimates of Circle Overlap (a) and Average Mutual Information in wrist-yaw rotation (b) across task difficulty conditions. Connected arrows represent significant pair-wise differences at $p<.05$. Error bars are standard errors.

Finally, Pearson $(r)$ correlations were used to assess the relationship between team performance (i.e., Circle Overlap) and nonlinear wrist coupling strength for each task difficulty condition (Table 2). Interestingly, all of the nonlinear metrics we employed were analogous in detecting a negative relationship between PBC and performance across all conditions, indicating that a decrease in coupling was related to better performance. In other words, lower similarity in the temporal patterning of coupled wrist movements was related to a greater ability to keep the two circles aligned. The similar direction of effect detected across measures is also intriguing, and suggests that these measure, though computational quite different form one another, are sensitive to similar dynamical properties about a coupled relationship (as argued in section 1.2).

Still, there were noticeable differences in the magnitude of relationship detected between wrist coupling and team performance across measures. Specifically, \%REC detected the strongest relationship $(r=-.50$, collapsed across condition and rotational plane), followed by $\operatorname{CSEn}^{-1}(r=-.40)$ and AMI $(r=-.26)$, respectively. This may indicate sensitivity differences between these measures, at least in-so-far as accounting for individual differences in team performance are concerned.

Table 2. Pearson correlations between PBC measures and team performance (Circle Overlap) for each task difficulty condition

\begin{tabular}{|c|c|c|c|}
\hline \multirow[b]{2}{*}{ PBC Measure } & \multicolumn{3}{|c|}{$\underline{\text { Task Difficulty Condition }}$} \\
\hline & Easy & Normal & Hard \\
\hline CSEn $^{1}$ - yaw & $-.38^{*}$ & $-.54^{*}$ & -.25 \\
\hline CSEn $^{1}$ - pitch & $-.48^{*}$ & $-.41^{*}$ & -.33 \\
\hline$\%$ REC - yaw & $-.51^{*}$ & -.31 & $-.56^{\star}$ \\
\hline$\%$ REC - pitch & $-.41^{*}$ & $-.68^{*}$ & $-.50^{\star}$ \\
\hline AMI - yaw & $-.43^{*}$ & $-.40^{*}$ & -.23 \\
\hline AMI - pitch & -.32 & -.13 & -.03 \\
\hline
\end{tabular}




\subsection{Discussion}

Dyad wrist movements did not exhibit a linear, but rather a nonlinear coupled relationship in this experiment. This is important because it determined the family of statistics that were appropriate for examining PBC experimental effects, but also because it ruled out synchronicity (the coupling dynamic indexed by CC) as a coordination strategy that could have been utilized by teams to perform the task. However, we make this statement with some caution since our results do not exclude the possibility that a synchronized relationship might have existed at some temporal lag, potentially indicating a linear leader-follower coordination strategy. We are currently examining this issue.

Findings regarding the manipulated effect of task difficulty revealed lower nonlinear wrist coupling strength (reduced AMI) in conditions where the task was more difficult. In interpreting this effect, it is first important to mention that this finding does not insinuate that wrist movements were completely decoupled, since significant nonlinear coupling was confirmed through surrogation tests. Rather, this finding indicates that nonlinear coupling strength was simply less in higher difficulty conditions. Second, if the only other information provided is that performance was also decreased by increases in task difficulty, then one possible explanation is that higher difficulty may have inhibited the ability of teams to coordinate effectively, leading to decreased coupling and reduced performance. However, insight gained from the correlation analyses supports a different interpretation; namely, that a decrease in wrist coupling under higher task difficulty may have reflected a compensatory strategy. This interpretation is supported by the ubiquitous set of negative correlations detected between nonlinear wrist coupling and team performance across task difficulty conditions, indicating that decreases in wrist coupling strength were associated with increases in performance.

As mentioned in section 1.1, we found a similar relationship between team performance and PBC (in cardiac IBIs) in a previous study [5]. In that study, we posited that a negative correlation may have indicated general team coordination plasticity or a complimentary coordination strategy featuring asynchronous and/or anti-phase team member behaviors [25] - either of which could result in decreases in nonlinear coupling strength. Here we come to similar conclusions.

In considering sensitivity differences in the set of nonlinear measures we employed, it appears that our results lead to mixed interpretations. On one hand, because AMI was the only measure to detect meaningful changes in wrist coupling induced by the experimental manipulation of task difficulty, it could be argued that this metric was more sensitive than the others. However, \%REC exhibited the strongest correlations with performance, hinting that it was best in accounting for individual team performance differences. While the results of this study cannot definitively address issues of measure sensitivity across coupling metrics, they raise interesting possibilities. To formally address the issue further we have planned a series of modeling experiment wherein coupling strength will be mathematically manipulated, allowing for true quantitative comparisons. 


\section{Conclusion}

In this manuscript we presented an overview of PBC with a focus on studies that have explored its utility as an index of team processes and performance. In addition, we provided an overview of important measurement issues in PBC research, followed by a simple empirical study that contextualized and accentuated this matter. Overall, we believe that examination of PBC is a fruitful area for ongoing research, since it not only appears to be informative for basic theory development of team dynamics but also has the potential for use in real-time team monitoring.

Acknowledgements. This research was generously supported by an Air Force Office of Scientific Research (AFOSR) grant (Program Manager: Dr. Jay Myung).

\section{References}

1. Knoblich, G., Butterfill, S., Sebanz, N.: Psychological research on joint action: Theory and data. In: Ross, B. (ed.) The Psychology of Learning and Motivation. Academic Press, Burlington (2011)

2. Henning, R.A., Boucsein, W., Gil, M.C.: Social-physiological compliance as a determinant of team performance. Int. J. Psychophysiol. 40, 221-232 (2001)

3. Elkins, A.N., Muth, E.R., Hoover, A.W., Walker, A.D., Carpenter, T.L., Switzer, F.S.: Physiological compliance and team performance. Appl. Ergon. 40, 997-1000 (2009)

4. Shockley, K., Baker, A.A., Richardson, M.J., Fowler, C.A.: Articulatory constraints on interpersonal postural coordination. J. Exp. Psychol. Human. 33, 201-208 (2007)

5. Strang, A.J., Funke, G.J., Knott, B.A., Warm, J.S.: Physio-behavioral synchronicity as an index of processes supporting team performance. Human Fac. Erg. Soc. P. 55, 1447-1451 (2011)

6. Salas, E., Fiore, S.M.: Team cognition:Understanding the factors that drive process and performance. American Psychological Association, Washington, DC (2004)

7. Konvalinka, I., Xygalatas, D., Bilbulia, J., Schjodt, U., Jegindo, E., Wallot, S., Van Orden, G., Roepstorff, A.: Synchronized arousal between performers and related spectators in a fire-walking ritual. P. Natl. A. Sci. USA 108, 8514-8519 (2011)

8. Bernieri, F.J.: Coordinated movement and rapport in teacher-student interactions. J. Nonverbal Behav. 12, 120-138 (1988)

9. Wiltermuth, S.S., Heath, C.: Synchrony and cooperation. Psycholog. Sci. 20, 1-5 (2009)

10. Mullen, B., Copper, C.: The relation between group cohesiveness and performance: integration. Psychol. Bull. 115, 201-227 (1994)

11. Gully, S.M., Incalcaterra, K., Joshi, A., Beaubien, J.M.: A meta-analysis of team-efficacy, potency, and performance: Interdependence and level of analysis as moderators of observed relationships. J. Appl. Psychol. 87, 819-832 (2002)

12. Shockley, K.: Cross recurrence quantification of interpersonal postural activity. In: Riley, M.A., Van Order, G.C. (eds.) Tutorials in Contemporary Nonlinear Methods for the Behavioral Sciences, http://www.nsf.gov/sbe/bcs/pac/nmbs/nmbs.j.sp (accessed February 1, 2013)

13. Schmidt, R.C., O'Brien, B.: Evaluating the dynamics of unintended interpersonal coordination. Ecol. Psychol. 9, 189-206 (1997) 
14. Peters, B.T., Haddad, J.M., Heiderscheit, B.C., Van Emmerick, R.E.A., Hamill, J.: Limitations in the use and interpretation of continuous relative phase. J. Biomech. 36, 271-274 (2003)

15. Chatfield, C.: The analysis of time series: An introduction, 6th edn. CRC Press, New York (2004)

16. Carroll, J.P., Freedman, W.: Nonstationary properties of postural sway. J. Biomech. 26, 409-416 (1993)

17. Zbilut, J.P., Webber, C.L., Zak, M.: Quantification of heart rate variability using methods derived from nonlinear dynamics. In: Drzewiecki, G.M., Li, J.K. (eds.) Analysis and Assessment of Cardiovascular Function, pp. 324-334. Springer, New York (1998)

18. Richman, J.S., Moorman, J.R.: Physiological time-series analysis using approximate and sample entropy. Am. J. Physiol-Heart C. 278, H2039-H2049 (2000)

19. Thomas, R.D., Moses, N.C., Semple, E.A., Strang, A.J.: A usable and efficient algorithm for the computation of average mutual information: Validation and implementation in Matlab. J. Math. Psycholog. (under review)

20. Pereda, E., Quiroga, R.Q., Bhattacharya, J.: Nonlinear multivariate analysis of neurophysiological signals. Prog. Neurobiol. 77, 1-37 (2005)

21. Shockley, K., Butwill, M., Zbilut, J.P., Webber, C.L.: Cross recurrence quantification of coupled oscillators. Phys. Lett. A 305, 59-69 (2002)

22. Liu, L.Z., Qian, X.Y., Lu, H.Y.: Cross-sample entropy of foreign exchange time series. Physica A 389, 4785-4792 (2010)

23. Zhang, T., Yang, Z., Coote, J.H.: Cross-sample entropy statistic as a measure of complexity and regularity of renal sympathetic nerve activity in the rat. Exp. Physiol. 92, 659-669 (2007)

24. Ramdani, S., Seigle, B., Lagarde, J., Bouchara, F., Bernard, P.L.: On the use of sample entropy to analyze human postural sway data. Med. Eng. Phys. 31, 1023-1031 (2009)

25. van Schie, H.T., Waterschoot, B.M., Bekkering, H.: Understanding action beyond imitation: Reversed compatibility effects of action observation in imitation and joint action. J. Exp. Psychol. Human. 34, 1493-1500 (2008) 